\title{
Special issue: Innovative Decision Systems, extended papers from the 12th EANN/7th IFIP AIAI 2011 Joint Conferences
}

\author{
Ilias Maglogiannis ${ }^{\mathrm{a}, *}$, Lazaros Iliadis ${ }^{\mathrm{b}}$ and Harris Papadopoulos ${ }^{\mathrm{c}}$ \\ ${ }^{a}$ University of Central Greece \\ ${ }^{\mathrm{b}}$ Democritus University of Thrace, Greece \\ ${ }^{\mathrm{c}}$ Frederick University, Cyprus
}

\begin{abstract}
In the new era of computational intelligence, the ever-expanding abundance of information storage and processing power enables researchers and users to tackle applications on various scientific domains of Decision Support Systems (DSS). The general focus of this special issue is to provide insights on how computational intelligence methodologies and algorithms can be employed in real world applications, so as to produce information systems capable of solving important and hard decision problems. This special issue on "Innovative Decision Systems" contains extended versions of seven (7) papers selected from the 12th EANN/7th AIAI 2011 Joint Conference. The manuscripts were accepted for publication, after passing through a peer review process by at least two independent academic referees. AIAI and EANN are two well-established annual events, technically sponsored by the Technical Committee 12, Working Group 12.5 (TC12-WG12.5) of the International Federation for Information Processing (IFIP) and the International Neural Network Society (INNS) respectively. The 2011 event was held on September 15-18 2011 in Corfu, Greece.
\end{abstract}

\section{Introduction}

The papers included in this special issue cover a range of topics, addressing various distinct domains of decision technologies. The application areas include biomedical informatics, project and portfolio management, market basket analysis, home automation and software cost estimation.

More specifically in the medical informatics domain Loizou et al. study the application of standard classification methods for the prognosis of future disability in subjects diagnosed with clinical isolated syndrome (CIS) of multiple sclerosis (MS). Several image texture analysis features were extracted from brain MR imaging scans of patients. Authors have identified the features that could give acceptable significant differ-

\footnotetext{
${ }^{*}$ Corresponding author. Ilias Maglogiannis, University of Central Greece. E-mail: E-mail: imaglo@ucg.gr.
}

ence using the Wilcoxon test. Based on these features three different classification models were investigated for predicting a subject's disability score. The utilized classification models were Probabilistic Neural Networks (PNN), Decision Trees (C4.5) and Support Vector Machines (SVM), which achieved the highest percentage of correct classification's score.

In the biomedical field also, Valavanis et al. deal with the prediction of Obstructive Nephropathy (ON), which is a renal disease with pathological profile resulting in tightly coupled and co-regulated, molecular processes. Authors present in their paper an intelligent workflow for predictive analytical purposes, on an integrative ON dataset, encompassing human micro RNA (miRNA) microarray data and mouse orthologous messenger RNA (mRNA) microarray data. The workflow is implemented in Rapidminer, an open access data mining and predictive analysis platform. The scope of this work was (i) the selection of the most reliable predictive biomarkers and (ii) the assessment of 
their classification power for discriminating ON severity related classes.

Similarly in the field of wellness and homecare Vazquez et al. describe the application of a global intelligent decision system for smart homes in the field of air quality and thermal comfort management. The proposed approach faces the high heterogeneity of smart homes by means of an extensive knowledge base and a cooperative framework of intelligent agents. On

this structure, the underlying methodology identifies users' habits and behaviors as the main common elements that join home applications, services and technologies in a sound overall performance.

Milios et al. in their paper deal with the field of software cost estimation. More specifically the authors discuss the Estimation by Analogy method (or EbA); a case-based reasoning technique, in which cost estimation is produced by comparing the characteristics of the target project with those of old cases. The authors propose an optimal global setup for determining empirically the best parameter configuration of EbA based on genetic algorithms. Authors report results on two datasets and compare with approaches that explore partially the search space.

Stylianou and Andreou are handling the software project management domain aiming at the development of a decision system for scheduling projects and forming teams in the best possible way given a set of tasks and developers. The approach employs a multiobjective genetic algorithm to optimize various aspects of scheduling and staffing in the form of objective functions with respect to project duration and developer skills and at the same time handling constraints concerning task dependencies and assignment conflicts. The proposed methodology is assessed using a set of scenarios of varying project size and complexity that depict possible real-world software project cases.

Papavasileiou and Tsadiras deal with the market basket analysis problem in order to discover association rules between products in retail markets. More specifically, they propose a method of identifying the most valuable association rules based on indicators, concerning a) the variability of an association rule, and b) the variability of a product. Their method identifies the most inflexible association rules (rules that present low degree of variability) and provides the most valuable association rules regarding consumer behavior. The above method is applied to annual customer transaction data that have been collected by the use of loyalty cards from a known Greek supermarket chain.
Gerogiannis et al. present a Project and Portfolio Management Information System (PPMIS) selection/evaluation approach that applies a hybrid group decision-making method based on TOPSIS and Intuitionistic Fuzzy Sets (IFS). The approach considers the vagueness of assessors' judgments when evaluating PPMIS and the uncertainty of users when they judge their needs. The approach is demonstrated through a case study aiming to support the Hellenic Open University to select a suitable PPMIS.

The papers included in this special issue give a good picture of some innovative decision systems concerning a spectrum of application domains. We strongly believe that they are of high interest for the scientists of the intelligent decisions technology community and we deliver them to our readers with the hope that they will trigger further investigations and more applications towards the solution of real life problems.

Ilias Maglogiannis University of Central Greece, Secretary of the IFIP Working Group WG12.5.

Lazaros Iliadis Democritus University of Thrace, Greece.

Harris Papadopoulos Frederick University, Cyprus. (Guest Editors).

\section{Acknowledgements}

The co-editors would like to thank the following referees for their valuable help and support during the reviewing processes.
List of Referees
Ioannis Anagnostopoulos
Giorgos Anastassopoulos
Andreas Andreou
Charalampos Doukas
Efstratios Georgopoulos
Lazaros Iliadis
Jacek Kabzinski
Efthyvoulos Kyriacou
Spiridon Likothanassis
Ilias Maglogiannis
Harris Papadopoulos
Efi Papatheocharous
George Potamias
Athanasios Tsadiras
George Vouros 